\title{
Del modelo biomédico a la salud renal poblacional. Un cambio paradigmático imprescindible
}

\section{From the biomedical model to population kidney health. An essential paradigm shift}

\author{
Santos Ángel Depine ${ }^{(\mathbb{D})}{ }^{1,2} \square$ \\ ${ }^{1}$ Grupo de Investigación en Nefrología, Facultad de Ciencias de la Salud, Universidad Simón Bolívar, \\ Barranquilla, Colombia. \\ ${ }^{2}$ Dirección de Calidad, Confederación de Asociaciones de Diálisis de la República Argentina, Ciudad Autónoma \\ de Buenos Aires, Argentina.
}

Cómo citar: Depine SA. Del modelo biomédico a la salud renal poblacional. Un cambio paradigmático imprescindible. Rev. Colomb. Nefrol. 2021 8(3), e576. https://oi.org/10.22265/acnef.8.3.576

La enfermedad renal crónica (ERC), como exponente de las vulnerabilidades sociales, nos desafía a generar un cambio profundo en las políticas sanitarias, por lo cual a nivel mundial se viene trabajando en mejorar el abordaje de esta patología, así como en solucionar las importantes implicaciones que trae consigo en los pacientes que la padecen y en la sociedad en general.

No obstante, cuando los datos de la situación actual de esta enfermedad se consolidan en tablas estadísticas encontramos cifras desalentadoras que nos entristecen y preocupan, pues demuestran que lo realizado hasta ahora no ha sido suficiente ni totalmente efectivo.

En 2017 se demostró que 1,2 millones de personas murieron por ERC a nivel mundial, y que además en el período 1990-2017 se presentó un aumento del 41,5\% en la tasa global de mortalidad por dicha enfermedad. De esta forma, la ERC pasó de ser la decimoséptima principal causa de muerte en todo el mundo en 1990, a ocupar el decimosegundo lugar en 2017 [1].

$\square$ Correspondencia: adepineintramed.net. 
Los datos también muestran que entre 1990 y 2017 se registró un crecimiento del 29,3\% en la prevalencia global de la ERC en todas las edades, a lo cual se suma una preocupación adicional por los 35,8 millones de años de vida perdidos, los cuales son medidos por los indicadores de años de vida ajustados por discapacidad (AVAD), que a su vez combinan los años potenciales de vida perdidos con los años vividos con discapacidad dentro del proceso salud-enfermedad [1].

Es importante mencionar que establecer las cifras de mortalidad prematura y discapacidad asociada a las enfermedades permite medir las pérdidas de salud deuna población [2]. Además, cuando se establecen cuáles son los estratos sociales a los que más pertenecen los pacientes con ERC, se observa que la mayoría están concentrados en los tres quintiles más bajos del índice sociodemográfico, aunque es de destacar que todos los quintiles de este índice mostraron un aumento neto en el número absoluto de AVAD atribuibles a la ERC entre 1990 y 2017 [1].

No existen dudas de que la pobreza, entendida como la "privación o la falta de acceso a los medios a través de Las evidencias muestran los cuáles las personas pueden materializar plenamente su potencial humano" [3], y las condiciones de aislamiento social de muchas poblaciones en desventaja, como los pueblos originarios alejados de la visibilidad sanitaria [4], condicionan a las personas a enfermarse, pues estas no la profunda inequidad existente en diferentes regiones del planeta como Latinoamérica pueden desarrollar conductas saludables que en general implican disponer de capacidad económica y acceso a los servicios de salud. De igual forma, estas poblaciones tienen barreras para acceder al cuidado de la salud dadas por sus atavismos culturales y por la influencia de los chamanes, curanderos, hechiceros o brujos, quienes suelen ser la única alternativa de atención en salud en algunas áreas de países en desarrollo.

Al respecto, Abdullahi [5] establece que la proporción de curanderos por habitantes en África es de 1:500, mientras que la de médicos por habitantes es de 1:40.000, lo que explica porqué para millones de personas de las zonas rurales los curanderos siguen siendo sus principales, o incluso únicos, dispensadores de atención sanitaria [5].

Las evidencias muestran la profunda inequidad existente en diferentes regiones del planeta como Latinoamérica [6] y, además, ponen de manifiesto las fallas de las políticas públicas orientadas a esas poblaciones vulnerables, en las cuales, a parte de las carencias de los aspectos sanitarios, es frecuente observar un muy bajo nivel educativo que también incide en la inaccesibilidad a los centros de salud existentes; esta última situación muchas 
veces predomina sobre las dificultades de accesibilidad geográfica. En este sentido, la estrecha relación entre educación y salud demuestra que los adultos que poseen un mayor nivel educativo tienen una mejor salud y esperanza de vida [7].

Otro condicionante que puede transformarse en determinante para agigantar la desigualdad en la atención en salud y, de manera simultánea, favorecer el desarrollo de ERC en algunas regiones es la coexistencia de la pobreza con hábitats de vivienda en ambientes tóxicos de diferentes etiologías y procedencias, ya sea por contaminantes del agua para consumo [8], del suelo [9] o del aire. Al respecto, la Organización Panamericana de la Salud [10] informa que, en todo el mundo, cerca de 7 millones de muertes prematuras se atribuyeron a la contaminación del aire ambiental en 2016, y que alrededor del $88 \%$ de estos decesos ocurrieron en países de ingresos bajos y medios.

Dadas las tasas de crecimiento de la ERC; los años perdidos por discapacidad o muerte prematura; la falta de acceso al cuidado de la salud de grandes conglomerados de población, y la gran cantidad de personas que no han podido acceder a los tratamientos de remplazo de la función renal y han fallecido en estadios previos de la enfermedad, es evidente que las políticas sanitarias actuales de prevención y atención de las enfermedades renales basadas en factores de riesgo individuales no han sido efectivas ni eficientes, por lo cual es necesario repensar los modelos actuales y generar propuestas holísticas e integradoras.

Por otra parte, en los últimos años en algunas regiones se ha registrado una alarmante presencia de pacientes con ERC de etiología desconocida y características epidémicas, como Otro gran avance ha cual se estableció como una ERC de causas no tradicionales sido el de la medicina de presente en Centroamérica e India, incluida Sri Lanka, y precisión que se acompaña de una elevada mortalidad en personas jóvenes (menores de 60 años); esta entidad ha generado inconvenientes asistenciales en algunas comunidades por la saturación de la capacidad instalada especializada, la cual no está preparada para atender una alta carga de población enferma.

El diagnóstico anatomopatológico de la nefropatía de comunidades agrícolas es el de una nefritis intersticial crónica cuyo origen se considera multicausal, y entre las causas se menciona el estrés térmico y algunos plaguicidas específicos; de igual forma, no se descartan los factores genéticos y la presencia de agentes infecciosos, además en una proporción importante de los casos se da un gran protagonismo a los aspectos sociales vulnerables en 
que viven las poblaciones afectadas [11].

De igual forma, en la búsqueda de mejores resultados para el seguimiento de pacientes con enfermedad renal se han incorporado algunas prácticas personalizadas de atención, como es el caso del programa de navegadores de pacientes, el cual tiene la finalidad de involucrar a cada paciente y a su equipo de atención para que sean proactivos y se encuentren mejor preparados para dar respuesta a la evolución de la enfermedad de acuerdo con los lineamientos y principios del modelo de atención crónica; estas prácticas generan pacientes informados y activos [12].

Otro gran avance ha sido el de la medicina de precisión, pues los análisis de secuenciación han identificado numerosas variantes genéticas relacionadas con enfermedades renales específicas, generalmente aquellas de manifestación temprana en la vida y causadas por mutaciones de un solo gen [13]. Sin embargo, su gran inconveniente radica en la complejidad genética y fenotípica de las enfermedades renales, por lo cual es necesario establecer cómo interactúan las cuestiones genéticas de estas patologías con las exposiciones ambientales, lo que también permitiría definir molecularmente algunos subgrupos y permitiría generar intervenciones terapéuticas específicas [14,15].

Todos estos adelantos, que se suman a otros vinculados a las mejores prácticas asistenciales, no han cubierto el amplio espectro epidemiológico de la ERC en el mundo, ni tampoco han mitigado sus graves consecuencias humanas y económicas, por lo que es necesario seguir trabajando en el tema.

Latinoamérica ha sido precursora en establecer un modelo de salud renal $[16,17]$, sin embargo es necesario repensar las estrategias implementadas hasta ahora y considerar una articulación transdisciplinaria y holística en un nuevo modelo con características poblacionales y que integre factores de riesgo individuales, factores de riesgo de la población y sus indicadores, incluidos los determinantes sociales que se vinculan a la salud de las comunidades. De esta forma, y teniendo en cuenta que las políticas de salud poblacional pueden mitigar los efectos de los determinantes

\section{Es necesario seguir} trabajando en el tema sociales sobre la salud, es necesario que desde la academia y desde las sociedades científicas se otorgue preponderancia a esta visión integradora del proceso salud-enfermedad-atenciónrehabilitación-salud con el fin de facilitar la incorporación de la salud renal poblacional en los focos de interés de la salud pública y de la nefrología. 


\section{Referencias}

[1] GBD Chronic Kidney Disease Collaboration. Global, regional, and national burden of chronic kidney disease, 1990-2017: a systematic analysis for the Global Burden of Disease Study 2017. The Lancet. 2020;395(10225):709-33. https://oi.org/10.1016/S0140-6736(20) 30045-3. 个Ver página 1, 2

[2] Murray CJ, López AD. Evidence-based health policy-lessons from the Global Burden of Disease Study. Science. 1996;274(5288):740-3. https:/doi.org/10.1126/science.274.5288.740. $\uparrow$ Ver página 2

[3] Organización Mundial de la Salud (OMS). Influencia de la pobreza en la salud. Informe de la Secretaría. Ginebra: 113ª reunión Consejo Ejecutivo OMS; 2003 [citado junio 8 2021]. Disponible en: https:/apps.who.int/gb/archive/pdf_files/EB113/seb11312.pdf. $\uparrow$ Ver página 2

[4] Aroca-Martínez G, Cadena-Bonfanti A, Ardila-Cárdenas ME, Gonzáles-Torres HJ, LunaGonzález ML, Espítaleta-Vergara Z, et al. Día Mundial del Riñón 2019: determinación de factores de riesgo para enfermedad renal en indígenas adultos colombianos. Rev. Colomb. Nefrol. 2020;7(Supl):8-17. 个Ver página 2

[5] Abdullahi AA. Trends and Challenges of Traditional Medicine in Africa. Afr J Tradit Complement Altern Med. 2011;8(5 Suppl): 115-123. https:/dx.doi.org/10.4314/ajtcam.v8i5S.5. $\uparrow$ Ver página 2

[6] Depine SA, Aroca-Martínez G. Desafiando a la inequidad de Latinoamérica. estrategias facilitadoras de çontrol"de la Enfermedad Renal Crónica. Barranquilla: Ediciones Universidad Simón Bolivar; 2018. ^Ver página 2

[7] Raghupathi V, Raghupathi W. The influence of education on health: an empirical assessment of OECD countries for the period 1995-201. Arch Public Health. 2020; 78(20). https://doi.org/10.1186/s13690-020-00402-5. 个Ver página3

[8] Robles-Osorio M, Sabatha E. Disparidad social, factores de riesgo y enfermedad renal cró-

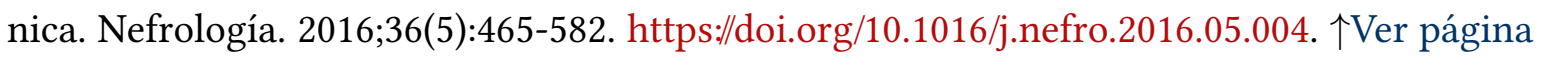
3

[9] Food and Agriculture Organization of the United Nations (FAO). Proceedings of the Global Symposium on Soil Pollution 2018. Rome: FAO; 2018. $\uparrow$ Ver página 3

[10] Organización Panamericana de la Salud .OPS). Calidad del aire. Nueva York: OPS; 2018

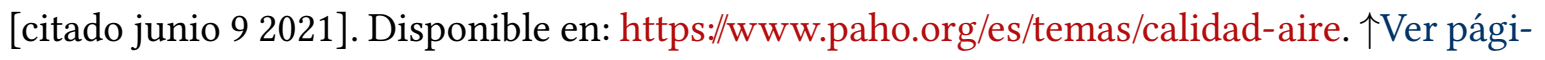
na 3 
[11] Hoy W, Ordunez P, editores. Epidemia de enfermedad renal crónica en comunidades agrícolas de Centroamérica. Definición de casos, base metodológica y enfoques para la vigilancia de salud pública. Washington: Organización Panamericana de la Salud; 2017. 个Ver página 4

[12] Freund KM. Patient Navigation: The Promise to Reduce Health Disparities. J Gen Intern Med. 2011;26(2):110-2. https://doi.org/10.1007/s11606-010-1593-5. 个Ver página 4

[13] Vivante A, Hildebrandt F. Exploring the genetic basis of early-onset chronic kidney disea-

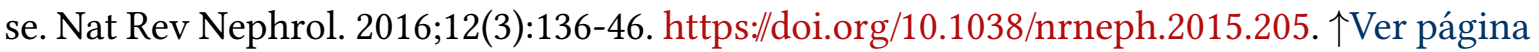
4

[14] Wyatt CM, Schlondorff D. Precision medicine comes of age in nephrology: identification of novel biomarkers and therapeutic targets for chronic kidney disease. Kidney Int. 2016;89(4):732-7. https://doi.org/10.1016/j.kint.2016.02.009. 个Ver página 4

[15] Precision medicine in nephrology. Nat Rev Nephrol. 2020;16(1):615. https://oi.org/10. 1038/s41581-020-00360-9. 个Ver página 4

[16] Burgos-Calderón R, Depine S. Sustainable and tenable renal health model: A Latin American proposal of classification, programming, and evaluation. Kidney Int Suppl. 2005;(Suppl

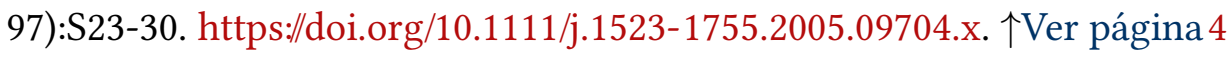

[17] Depine S, Burgos-Calderón R. Renal Health Models in Latin America: Development of National Programs of Renal Health. Ren Fail. 2006;28(8):649-64. https://doi.org/10.1080/ 08860220600925743. $\uparrow$ Ver página 4 